By not observing the fundamental difference between rules and laws, much confusion has already been created. We do not see how Genetics will be able to deserve the place among the inductive sciences it behoves it to take, so long as whole groups of people, studying the behaviour of characters in crosses continue to ignore such fundamental things.

Bussum (Holland), September I9I3.

\title{
Notiz über den Begriff der Kryptomerie.
}

\author{
Von Professor Dr. Erich v. Tsehermak.
}

(Eingegangen: 28. September I9I3.)

Die Feststellung eines regelmäBigen, und zwar in Mendelschen Zahlenverhältnissen erfolgenden Auftretens gewisser Bastardierungsneuheiten oder wahrer Hybridnova - nicht bloßer Neukombinationen von Merkmalen, die bereits an den Stammeltern ausgeprägt waren, hat mich im Jahre I903 dazu geführt, den Begriff der Kryptomerie aufzustellen und denselben mit Absicht zunächst rein experimentellerfahrungsmäßig $\mathrm{zu}$ fassen ${ }^{1}$ ). Ich wollte die neugewonnene Arbeitsrichtung und mich selbst nicht von vornherein auf eine bestimmte Spezialerklärung oder Theorie der beobachteten Erscheinungen festlegen.

Die damals gegebene Definition bezeichnete als kryptomer ,,solche Formen, welche bei Inzucht in bestimmten charakteristischen Merkmalen konstant sind, jedoch im AnschluB an Fremdbestäubung ohne Zufuhr eines neuen bezüglichen Merkmales dennoch eine charakterische Abänderung jener Merkmale, also neue Eigenschaften oder „Kreuzungsnova" zutage treten lassen". Die bei Fremdkreuzung entweder schon in $F_{1}$ oder in $F_{2}$ gesetzmäßig auftretenden Nova wurden von mir in den einfachsten Fällen als dominierend $\left(\mathrm{F}_{2} 9: 3: 4\right.$ oder $\left.9: 7\right)$, mit-

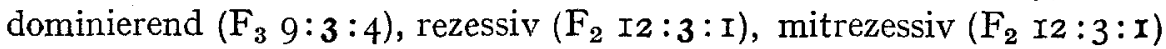
bezeichnet ${ }^{2}$ ). Bei der damaligen Fassung des Begriffes der Kryptomerie spielte vorwiegend noch die Vorstellung mit, da 3 die fremde Form

1) Die Theorie der Kryptomerie und des Kryptohybridismus I. Mitt. Uber die Existenz kryptomerer Pflanzenformen. Beitr. z. Bot. Zentrbl. Bd. I6 H. I S. I-25 I903.

2) Zitat aus 1903. Vgl. auch: Weitere Kreuzungsstudien an Erbsen, Levkojen und Bohnen. Zeitschr. f. d. landw. Versuchsw. in Osterreich I904, S. I-I06. 
nur ein auslösendes Agens, einen Aktivator oder ein sogenanntes Komplement abgebe, da 3 also in der kryptomeren Form eine eigentliche Anlage zu einer bestimmten Eigenschaft latent vorhanden sei, welche nur der Aktivierung bedürfe. Die beiden Formen, deren Bastardierung ein reguläres Novum ergibt, wurden also als in ungleichem $\mathrm{Maße}$ an der Erzeugung des Novums beteiligt angesehen, wofür speziell der Umstand maßgebend war, daß einerseits alle oder fast alle geprüften Rassen von Pisum sativum sich befähigt erwiesen, mit einzelnen Rassen von Pisum arvense Nova zu ergeben, und da $B$ andererseits analoge Nova anscheinend spontan auch in scheinbar reingezüchteten Beständen von Pisum arvense auftraten - beispielsweise gelegentliche Rotblüte oder Samenschalenpunktierung unter sonst konstanten Beständen von rosablühenden oder punktlosen Erbsen.

Analoge Bastardierungsnova, wie ich sie in den oben zitierten Mitteilungen beschrieb, wurden von anderen Autoren in großer Zahl festgestellt - so von Cuénot, Correns, Shull, Bateson mit Saunders und Punnett. Die von den ebengenannten ausgebildete Faktorentheorie, deren Grundidee bekanntlich auf Gregor Mendel zurückgeht, wurde mit Erfolg gerade zur Erklärung solcher mendelnder Hybridnova angewendet. Ich konnte mich dazu erst entschließen, nachdem ich in jahrelangen Versuchen die Faktorenlehre einer eingehenden und vielseitigen Prüfung unterworfen hatte, indem ich die Hybriddeszendenten systematisch neuerlichen Bastardierungen, und zwar mit ihren Stammeltern sowie untereinander, ferner mit reinen fremden Formen sowie mit Hybriddeszendenten anderer Herkunft unterzog und das experimentelle Resultat mit der aus der Faktorenlehre abgeleiteten Erwartung verglich $\mathbf{1}$ ). Die dabei bewährt gefundene und ganz wesentlich erhärtete Faktorenlehre erwies sich auch als zureichend für die Erklärung des zahlenmäßigen Verhaltens all der von mir beobachteten Bastardierungsnova. Dieselben ließen sich teilweise auf eine Synthese, teilweise auf eine Analyse von Faktoren (Bateson) zurückführen. Speziell fanden die früher erwähnten einfachsten Fälle eines dominierenden, mitdominierenden, rezessiven oder mitrezessiven Novums eine Erklärung als Fälle von bifaktoriellem Unterschied oder

1) Vgl. bereits meine Darstellung der innerlichen oder wesentlichen Vererbungsweise nach der Faktorenlehre in Bd. IV der Züchtung der landwirtschaftlichen Kulturpflanzen von C. Fruwirth, 2. Aufl. igio S. 88-105 und speziell: Examen de la théorie des facteurs par le recroisement méthodique des hybrides. Paris IgI I sowie Bastardierungsversuche an Levkojen, Erbsen und Bohnen mit Rücksicht auf die Faktorenlehre. Zeitschr. f. indukt. Abst.- u. Vererbungslehre Bd. VII, H. 2, IgI 2. S. $81-234$. 
dihybrider Bastardierung - und zwar ließ sich die erste Gruppe zurückführen auf Zusammenfügung bisher getrennter Faktoren $(\widehat{\mathrm{AB}})$, die zweite Gruppe auf isoliertes Hervortreten eines bisher in Kombination $(\widehat{A B})$ gestandenen Faktors $(\mathrm{Ab})$ bei Wirkungslosigkeit des isolierten zweiten Faktors $(\mathrm{aB}=\mathrm{ab})$, die dritte Gruppe als isoliertes Hervortreten des bisher gehemmten, verdrängten oder verdeckten zweiten Faktors (aB) bei Gleicherscheinen aller die beiden Faktoren oder nur den ersten Faktor enthaltenden Formen $(\mathrm{AB}=\mathrm{Ab})$, endlich die vierte Gruppe als Hervortreten eines dritten Faktors im bisher nicht realisiert gewesenen Falle von Fehlen beider Faktoren (abC).

Die Art der Beziehung der einzelnen Faktoren bedurfte allerdings erst besonderen Studiums, welches mich dazu führte - zum Teil in Ubereinstimmung mit Nilsson-Ehle - ein bloB scheinbares und ein wirkliches Zusammenwirken von Faktoren zu unterscheiden, und zwar ein Zusammenwirken in gleichsinnig-positiver Richtung (Kombination ${ }^{1}$ ), Förderung) oder in gegensinnig-negativer Richtung (Hemmung, Verdrängung).

Die begreifliche Wertschätzung der hochbedeutsamen Faktorentheorie - welch erstere allerdings zum Teil schier an Uberschätzung grenzt ${ }^{2}$ ) - hat bei manchen Autoren dazu geführt, den Begriff der Kryptomerie in den Hintergrund treten zu lassen oder diesbezügliche Mißverständnisse aufkommen zu lassen. Die letzteren bestehen speziell in der Meinung, daB der Kryptomeriebegriff als überflüssig zu betrachten oder aufzulassen sei (Johannsen ${ }^{3}$ )) bzw. nichts anderes besage als latente Veranlagung zu einem bestimmten Merkmale (L. Plat $\left.\mathrm{e}^{4}\right)$ ). Es scheint mir daher angebracht, unter kurzer Bezugnahme auf meine eigenen neueren Beobachtungen einige aufklärende Bemerkungen $\mathrm{zu}$ machen und die neuere Fassung des Kryptomeriebegriffes darzulegen ${ }^{5}$ ).

1) Die seinerzeit ( 1910 ) von mir gemachte Spezialunterscheidung von Fällen bloßer Auslösung und von Fällen eigentlicher Kombination oder Synthese sei ausdrücklich aufgegeben angesichts des fließenden Charakters der Grenzen, und um nicht zu einer Minderbewertung der einen Komponente einer Kombination bzw. der einen Stammform eines Bastardierungsnovums zu verleiten.

2) Speziell gilt dies dann, wenn man die Analyse eines Bastardierungsfalles mit der Feststellung oder Annahme einer bestimmten Anzahl von Faktoren bereits erschöpft erachtet.

3) Johannsen, Elemente der Erblichkeitslehre. Jena 1909 S. 452 . Vgl. demgegenüber die Annahme und Verwertung des Kryptomeriebegriffes bei N. Heribert Nilsson, Die Variabilität der Oenotheya Lamarckiana und das Problem der Mutation. Zeitschr. f. ind. Abst.- u. Vererbungslehre Bd. VIII S. 89. I9I2.

4) L. Plate, Vererbungslehre. Handbücher der Abstanmungslehre. II. Bd. I9r3.

5) Vgl. meine kritischen Bemerkungen in der Arbeit ex 1912, S. 82, 227 und in der Besprechung von L. Plates Vererbungslehre. Wiener mediz. Wochenschrift Mai I9I 3. 
Im Geiste der von verschiedenen Seiten her gefestigten und erhärteten Faktorenlehre könnte man Kryptomerie ganz allgemein definieren als Besitz von Faktoren, welche bei geänderter Faktorenkonstellation - speziell bei Herstellung einer gleichsinnigen Beziehung (Zufuhr fremder Faktoren oder bloße "Assoziation" bereits vorhandener - siehe unten) oder bei Aufhebung einer gegensinnigen Beziehung (Abspaltung oder bloße Dissoziation) - in besonderer, neuartiger Weise wirksam zu werden vermögen. Kryptomerie im allgemeinsten Sinne bedeutet also Besitz von Faktoren von nichterschöpfter Wirksamkeit.

Zweckmäßiger erscheint es aber, diesen Begriff von der ganz allgemeinen Fassung einzuschränken auf die spezielle Bedingung, da $B$ der Besitz solcher reaktionsfähiger Faktoren sich äußerlich überhaupt nicht verrate, also ein unmerklicher sei. Die Ursache für die Unwirksamkeit der betreffenden Faktoren ist - in den zunächst betrachteten Fällẹn - gelegen in dem Mangel gewisser ergänzender Faktoren von gleichsinniger Wirkungsrichtung oder in dem gleichzeitigen Vorhandensein anderer Faktoren von gegensinniger Wirkungsrichtung. Eine Manifestation des kryptomeren Charakters bzw. der unwirksamen Faktoren ist demnach bei den einen kryptomeren Elementarformen erst möglich nach Zufuhr der "fehlenden“" Faktoren, bei den andern kryptomeren Elementarformen erst nach Abspaltung der gegensinnigen (hemmenden, verdrängenden, verdeckenden) Faktoren. Eine klare terminologische Unterscheidung aller solcher Formen von jenen, welche zwar äußerlich gleich erscheinen, jedoch in der inneren Zusammensetzung bzw. im Faktorengehalte, sei es durch Mangel oder durch Besitz, verschieden sind, erscheint mir absolut erforderlich $\mathbf{1}$ ). Es steht $\mathrm{zu}$ hoffen, da $B$ gerade Johannsen,

1) Um in einem Komplex äußerlich übereinstimmender Individuen die durch eventuelle Kryptomerie innerlich verschiedenen Elementarformen zu unterscheiden, genügt nicht ,eine Erprobung auf Konstanz unter Anwendung der besten Beobachtungsund Messungsmethoden, in einem Worte der Johannsenschen Methode (Linienprinzip)", wie dies P. Lotsy als praktisches Mittel zur Feststellung einer Elementarart (theoretisch zutreffend definiert als Summe gleichveranlagter homozygotischer Individuen) bezeichnet (Progr. rei botanicae, 4, Bd. S. 367, IgII), sondern es bedarf zudem der Erprobung durch vergleichende Bastardierung der einzelnen Individuen mit einer Anzahl geeigneter fremder Elementarformen. Nur wenn die einzelnen Individuen bei dieser Prüfung immer wieder übereinstimmende Bastardierungsprodukte, speziell übereinstimmend charakteristische Nova ergeben, ist ihre innere Zusammensetzung, ihre Faktorenformel als gleich zu betrachten. Man vergleiche speziell meine diesbezügliche Unterscheidung der einzelnen weißblühenden Elementarformen von Matthiola auf Grund vergleichender Bastardierungsprüfung (a. a. O. I912). 
welcher die begriffliche und terminologische Scheidung von äußerer Erscheinungsweise oder Phaenotypus und innerer Zusammensetzung oder Genotypus so klar und verdienstvoll erfaßt hat, nach dieser Klarstellung, derzufolge Kryptomerie im engeren Sinne den unmerklichen Besitz reaktionsfähiger Faktoren bedeuten soll, und zwar unter Wirkungsbehinderung durch Fehlen oder unter Wirkungsbehinderung bzw. Wirkungsablenkung durch Vorhandensein gewisser anderer Faktoren, mir beipflichtet. - Bei einer Mehrzahl von Falktoren, vor denen jeder für sich, aber auch gewisse Kombinationen unmerklich bleiben, resultieren bei der Aufspaltung mehrere verschiedene kryptomere Formen, welche die Verschiedenheit ihrer inneren Zusammensetzung bei Rehybridisation deutlich verraten. So konnte ich, wie bereits Igro mitgeteilt und IgI2 im Detail belegt, aus der Bastardierung rote behaarte $\times$ bestimmte weiBe glatte Levkoje ( $\mathrm{AbC}$ bzw. $\mathrm{A}_{1} \mathrm{~A}_{2} \mathrm{~A}_{3} \mathrm{bC} \times \mathrm{aBc}$ bzw. $\mathrm{a}_{1} \mathrm{~A}_{2} \mathrm{~A}_{3} \mathrm{Bc}$ ) die theoretisch deduzierbaren viererlei homozygotischen $W e i B(a \mathrm{BC}, \mathrm{aBc}, \mathrm{abC}, \mathrm{abc})$ unter den Spaltungsprodukten tatsächlich isolieren. Drei davon sind als kryptomer bezüglich der Faktoren BC oder B oder $\mathrm{C}$ zu bezeichnen; nur die vierte Form (abc) ist nicht mehr kryptomer. Die Folge jener Kryptomerie ist es, daß bei Rehybridisation, beispielsweise mit Aschrosa (Abc), in dem ersten Falle $(\mathrm{aBC} \times \mathrm{Abc})$ eine Reinviolette, im zweiten $(\mathrm{aBc} \times \mathrm{Abc})$ eine aschviolette, im dritten $(\mathrm{abC} \times \mathrm{Abc})$ eine reinrosa, im vierten $(a b c \times A b c)$ eine aschrosa $F_{1}{ }^{\prime}$-Generation resultiert. Andererseits sind nicht-kryptomere Hybriddeszendenten vom Pisum sativum-Typus aus Kreuzungen $P$. sativum $\times P$. arvense zu extrahieren $\left.{ }^{1}\right)-$ beispielsweise solche von der Formel ab aus rosa $(\mathrm{Ab}) \times$ weiB $(\mathrm{aB})$, cd aus makellos $P$. arvense $(\mathrm{Cd}) \times$ makellos $P$. sativum (cD), ef aus punktlos $P$. arvense (Ef) $\times$ punktlos $P$. sativum ${ }^{2}$ ) ( $\mathrm{eF}$ ).

Aus dem Dargelegten ist zugleich zu ersehen, daß Kryptomerie - zumal in der seit I9ro von mir gegebenen Begriffsbestimmung und Besitz einer latenten Anlage $\mathrm{zu}$ einem bestimmten Merkmal keineswegs gleichzusetzen sind, wie dies L. Plate meint. Der Begriff einer solchen Latenz entspricht überhaupt nicht mehr den Vorstellungen, zu welchen uns die Ausgestaltung und die Verifizierung der Faktorenlehre geführt hat. Jener Begriff erfaßt die Latenz von Faktoren - nur von einer solchen könnte man sprechen - unzweckmäßigerweise vom Gesichtspunkte der äußeren Wirkung, des Phaeno-

1) Vgl. a. a. O. I9I2 S. I $44, \mathrm{I} 54, \mathrm{I} 56$.

2) Eine Ausnahme gegenüber den gewöhnlichen kryptomeren (eF) $\dot{P}$. sativumRassen bildet die nichtkryptomere Rasse "Victoria mit violettem Nabel" (ef). 
typus (nach Johannsen) aus und verleitet leicht zur Minderbewertung des zur Manifestation oder „Aktivität" führenden Faktors. Demnach liegt mit obiger Gleichsetzung von Kryptomerie und Anlagenlatenz ein augenscheinliches Mißverständnis L. Plates vor.

Eine besondere Spezialbedeutung hat der Kryptomeriebegriff endlich gewonnen durch seine Anwendung auf solche Elementarformen, in welchen gleichfalls reaktionsfähige Faktoren unmerklich vorhanden sind, ihre Wirkungslosigkeit jedoch nicht - wie bei den bisher erörterten Fällen von Kryptomerie sensu strictiori - durch Fehlen oder durch Vorhandensein gewisser anderer Faktoren bedingt ist, sondern auf Unterbleiben einer Wechselwirkung oder Assoziation bereits vorhandener Faktoren beruht. Solche Elementarformen oder Individuen seien als dissoziativ-kryptomer bezeichnet. Die Ursache für das Ausbleiben der typischen Assoziation trotz des Nebeneinandergegebenseins der zur Reaktion erforderlichen Komponenten ist zunächst nicht aufzeigbar. Das Hindernis könnte physikalisch-chemischer Natur $\operatorname{sein}^{1}$ ).

Es ist keineswegs ausgeschlossen, da $B$ gewisse innere Momente, die nicht durch eine Bastardierung bzw. durch Heterozygotie gegeben sind, aber möglicherweise auch gewisse noch nicht näher bezeichenbare äußere Umstände (klimatischer, thermischer, traumatischer Natur wie auch bestimmte Kulturbedingungen) die Reaktionsbedingungen zwischen den Faktoren einer dissoziativ-kryptomeren Form in der Weise zu beeinflussen vermögen, da $B$ plötzlich an einem und dem andern Individuum, ja an einem ganzen Stamm Assoziation eintritt und damit sprunghaft ein neues Merkmal zutage tritt. Allerdings kann ich in den von mir beobachteten Fällen keinen äußeren Grund für die gelegentliche Assoziation anführen, muß also diese Fälle als solche von ,,spontaner" Assoziation bezeichnen. Eine solche Entstehung einer scheinbar neuen, eventuell weiterhin völlig konstanten Elementarform durch bloße Assoziation bisher kryptomer vorhandener Faktoren - ohne daB eine Bastardierung bzw. eine Zufuhr neuer Faktoren stattgefunden hätte - bewirkt den Anschein einer Mutation. Ihre Herkunftsweise wird erst klar durch Feststeilung und Berücksichtigung des dissoziativ-kryptomeren Charakters der Ausgangsform, in welche die ,neue“ Form sehr wohl auch zurückschlagen könnte.

J) Man vergleiche damit das gleichzeitige Vorhandensein von Glykogen und Diastase in der Leber und in den Muskeln der Winterfrösche, ohne daß Reaktion bzw. Zuckerbildung erfolgt. Hingegen erfolgt eine solche nach Aufhebung eines (etwa strukturellen) Hindernisses durch Zerreißen, Frieren und Auftauen (E. J. Lesser und 
Ganz analoge Möglichkeiten bestehen für Kryptomerie im Sinne einer bestehenden Hemmungsassoziation von Faktoren bzw. für eine dissoziative Mutation infolge Anfhörens einer solchen Beziehung. Es ergibt sich sonach eine neue Möglichkeit von sprunghafter Bildung neu erscheinender Elementarformen - nämlich durch eventuell nachdauernde Assoziation bzw. Dissoziation, d. h. durch Herstellung oder Lösung des Bandes zwischen vorhandenen Faktoren. Demgemäß resultiert meines Erachtens die Forderung einer gewissen Einschränkung der Thesen P. Lotsys ${ }^{1}$ ), daß Kreuzung der einzige Erwecker erblicher neuer Formen und die Ursache der Artbildung sei, indem nur dadurch neue Kombinationen von bereits vorhandenen Potenzen gebildet werden - ferner, da $B$ äußere Einwirkungen nicht zur Entstehung neuer Elementarformen führen können. Gewiß stimmt die Assoziations-Dissoziationstheorie insofern mit Lotsys Grundidee überein, als von beiden Seiten nicht eine Erzeugung neuer Faktoren angenommen wird. Bei der assoziativdissoziativen Neubildung von Elementarformen handelt es sich eben nicht um eine Änderung im Faktorenbestande bzw. in der Faktorenkombination an sich, sondern bloß um eine Änderung der Wirkungsweise vorhandener Faktoren aufeinander - um die Produktion verschiedener im Faktorenbesitz sozusagen isomerer Elementarformen. Gerade hiefür ist jedoch eine auslösende Einfrußnahme innerer Momente anzunehmen, die nicht mit Kreuzung zusammenhängen, sowie die allerdings noch unerwiesene Möglichkeit einer solchen Einflußnahme äußerer Momente keineswegs auszuschließen. Die exogenen Mutationen, wie sie Tower und Mac Dougall beschrieben haben, bedürfen jedenfalls einer Prüfung in diesem Sinne. - Andererseits muß der Unterscheidung verschiedener Arten von Variabilität nach Hagedoorn ${ }^{2}$ ) und Lotsy (a. a. O. S. 386) - nämlich

I. nichterbliche, exogene Modifikation,

2. erbliche Variation infolge Heterozygotie bzw. Mendelscher Spaltung,

J. Grade, Zeitschr. f. Biologie Bd. 60, S. 37I, 1913 sowie E. J. Lesser, Biochem. Zeitschr. Bd. 52, S. 47r, 19r3).

1) Versuche über Artbastarde und Betrachtungen über die Möglichkeit einer Evolution trotz Artbeständigkeit (Zeitschr. f. ind. Abst.- u. Vererbungsl. Bd. 8 S. 325-333 1912) und Fortschritte unserer Anschauungen über Deszendenz seit Darwin und der jetzige Standpunkt der Frage (Progr. rei botan. 4. Bd. S. 36I-388. I9I3).

2) Hagedoorn, Vorträge und Aufsätze über Entwicklungsmechanik der Organismen. Herausgegeben von W. Roux, Heft 12. Leipzig IgI2. 
3. erbliche Variation infolge Faktorenverlust bei der Gametenbildung (Verlustmutation - nach P. Lotsy noch fraglich) als neue Kategorie hinzugefügt werden:

4. erbliche Variation infolge Assoziation oder Dissoziation vorhandener Faktoren, wobei das betreffende Individuum entweder homozygotisch oder heterozygotisch ist. (Heterozygotie kann eine innere Bedingung für Assoziation sein, vgl. unten!)

Als dissoziativ-kryptomere Individuen bzw. Formen, welche sich durch fallweise Assoziation verrieten, konnte ich ${ }^{1}$ ) gewisse rosablühende Hybriddeszendenten aus Pisum sativum $\times P$. arvense feststellen, die als „rosa larvierte Rot-Individuen" anzusprechen sind (A $\downarrow B$ statt $\widehat{A B}-$ äußerlich gleich Ab). Analog sind dissoziativ-kryptomere ArvenseIndividuen ohne Samenschalenpunktierung, welche durch gelegentliches Auftreten punktierter Nachkommen den Besitz der Punktierungsfaktoren verraten $(E \downarrow F$ statt $\widehat{E F}-$ äußerlich gleich Ef oder eF oder ef vg1. I9I2 S. I57). Ein besonderes detailliertes Studium widmete ich dem dissoziierten Vorkommen des Marmorierungsfaktors neben dem Pigmentierungsfaktor bzw. den Pigmentierungsfaktoren in gewissen Rassen von Phaseolus vulgaris mit gleichfarbiger Samenschale (I9I2 S. I90-I95). Neben der dissoziativ-kryptomeren braunschaligen Rasse „Hundert für Eine“ (Abc $\downarrow \mathrm{M})$ erwiesen sich die meisten, aber nicht alle geprüften weißschaligen Rassen als kryptomer (aM) bezüglich des Marmorierungsfaktors, welcher sich übrigens in gewissen Bastardierungsfällen gleichfarbig $\times$ weiß nur bei Heterozygotie $(\mathrm{Mm})$ mit dem Pigmentfaktor assoziiert.

Auch für solche Merkmale, welche scheinbar einfache Mendelsche Vererbungsweise nach dem Pisum-Typus mit dem $\mathrm{F}_{2}$-Spaltungsverhältnis $3:$ I zeigen und daraufhin als unifaktoriell bewirkt betrachtet werden könnten - beispielsweise Behaarung gegenüber Glätte in den meisten Bastardierungsfällen von Matthiola -, besteht die Möglichkeit einer tatsächlichen bi- oder plurifaktoriellen Natur und damit einer Dissoziations-Kryptomerie. Ist nämlich die Verkoppelung von zwei oder mehr Faktoren eine absolute, so vererben sie sich wie ein einzelner Faktor: beispielsweise Faktor für Rotblüte A, erster Faktor für Blattachselmakel (C) und erster Faktor für Samenschalenpigmentierung $(G)$ und für Samenschalenpunktierung (E) in Pisum arvense - ebenso die chromogene Haupt- oder Grundkomponente $\left(A_{1}\right)$ und der Behaarungsfaktor bzw. die Behaarungsfaktoren $\left(T_{1} T_{2}\right)$ in behaarten weiBblühenden Levkojenrassen. Im Zusammenhange damit sei speziell

1) Vgl. a. a. O. I912, S. 145-I46. 
die Unterteilung des Pigmentierungs-, vielleicht auch des Behaarungsfaktors bei Matthiola in eine Mehrzahl von Einzelkomponenten betont. Ebenso wie bei gewissen Formen solche Einzelkomponenten doch getrennit vorkommen können, könnte bei anderen ein solcher Anschein erweckt sein nicht durch Fehlen gewisser Komponenten, sondern durch bloßes Ausbleiben einer Assoziation unter den vollzählig vorhandenen Komponenten. Gewisșe, z. B. äußere Umstände könnten ein solches Aufsplittern eines scheinbar einheitlichen Faktors in reaktionslose, dissoziierte Komponenten bewirken - ebenso wie andere Umstände ein Zusammenschweißen bisher aufgesplitterter Komponenten und damit beispielsweise Färbung, Behaarung u. dgl. hervorrufen könnten. Besonders für ein Hervorgehen von Kulturformen aus Wildformen ist, wie ich bereits betont ${ }^{1}$ ) und anderwärts detaillierter ausgeführt habe ${ }^{2}$, an eine solche Möglichkeit zu denken. Eine solche Vorstellung, daß bezüglich der einen Faktoren die Wildform, bezüglich anderer die Kulturform dissoziativ-kryptomer sei, erscheint gestützt. durch das gelegentliche, als Atavismus betrachtete Auftreten von dominierenden Wildformmerkmalen an rezessivmerkmaligen Kulturformen. Dasselbe kann sogar lokal in Form einer Knospenmutation erfolgen - beispielsweise konnte ich an Deszendenten aus Kreuzung verschiedener Kulturhaferrassen $\left(\mathrm{F}_{3}\right.$ Avena chinensis $\times$ Börstlösa Hafer, ebenso aus $\mathrm{F}_{3}$ Avena chinensis $\times$ Goldregen) gelegentlich inmitten eines. Ährchens vom Kulturhafertypus neben typischen Körnern vereinzelte solche vom Wildhafertypus (vollständig und dicht behaart, dunkelbraun mit knieförmig gebogener, kräftiger Granne und ringförmigem Kallus) beobachten. In solchen Fällen liegt es gewiß am nächsten, an eine gelegentliche Assoziation von dissoziiert in der Kulturform vorhandenen Wildformfaktoren zu denken bzw. die Kulturform als diesbezüglich dissoziativ-kryptomer zu betrachten. - Die Bedeutung, welche der von mir aufgestellten Theorie der Assoziation und Dissoziation von Faktoren für die Erklärung gewisser Atavismen und $\mathrm{Mu}$ tationen (einschließlich Knospenmutationen) zukommt, habe ich bereits an einem anderen Orte in den wesentlichen Zügen dargelegt, worauf ich hier nur zu verweisen brauche ${ }^{3}$ ).

1) E. v. Tschermak, Uber seltene Getreidebastarde. Beiträge zur Pflanzenzucht, 3. Heft S. 49-6I. Berlin I9I3.

2) E. v. Tschermak, Uber die Vererbungsweise bei Art- und Gattungsbastarden von Getreide. Vortrag bei der" Naturforscher-Vers., Sept. 19r3, Wien und Ober selteneGetreidebastarde. Beiträge zur Pflanzenzucht, Berlin, Parey I9I3- 3. Heft, S. 49-6I.

3) Diese Zeitschrift S. 228-230, 1912. 